\title{
Distributed Information-weighted Kalman Consensus Filter for Sensor Networks $^{\text {th }}$
}

\author{
Honghai Ji ${ }^{\mathrm{a}}$, Frank L. Lewis ${ }^{\mathrm{b}, \mathrm{c}}$, Zhongsheng Hou ${ }^{\mathrm{a}, 1}$, Dariusz Mikulski ${ }^{\mathrm{d}}$ \\ a Advanced Control Systems Lab, School of Electronics and Information Engineering, Beijing Jiaotong University, Beijing 100044, PR China \\ ${ }^{\mathrm{b}}$ UTA Research Institute, University of Texas at Arlington, Fort Worth, Texas, 76118, USA \\ ${ }^{\mathrm{c}}$ Qian Ren Consulting Professor, State Key Laboratory of Synthetical Automation for Process Industries, Northeastern University, Shenyang, 110819, PR \\ China \\ ${ }^{\mathrm{d}}$ U.S. Army Tank-Automotive Research Development \& Engineering Center
}

\begin{abstract}
Consensus-based algorithms for distributed Kalman filtering of the state of a dynamical target agent have attracted considerable research and attention during the past decade. In these filters, it is required for all agents to reach consensus about their estimates of the state of a target node. Distributed filtering techniques for sensor networks require less computation per sensor node and result in more robust estimation since they only use information from an agent's neighbors in a network. However, poor local sensor node estimates caused by limited observability, network topologies that restrict allowable communications, and communication noises between sensors are challenging issues not yet fully resolved in the framework of distributed Kalman consensus filters. This paper confronts these issues by introducing a novel distributed informationweighted Kalman consensus filter (IKCF) algorithm for sensor networks in a continuous-time setting. It is formally proven using Lyapunov techniques that, using the new distributed IKCF, the estimates of all sensors reach converge to consensus values that give locally optimal estimates of the state of the target. A new measurement model is selected that only depends on local information available at each node based on the prescribed communication topology, wherein all the estimates of neighbor sensors are weighted by their inverse-covariance matrices. Locally optimal solutions are then derived for the proposed distributed IKCF considering channel noises in the consensus terms. Moreover, if the target has a nonzero control input, a method is giving of incorporating estimates of the target's unknown input. Simulation case studies show that the proposed distributed IKCF outperforms other methods in the literature.
\end{abstract}

Key words: Multi-agent systems; Sensor networks; Information-weighted consensus; Distributed Kalman filters.

\section{Introduction}

This paper provides a novel algorithm for distributed Kalman filtering for multiple agents networked by a communication graph topology. In distributed filtering,

is This work is supported in part by the State Key Program (61433002), the Major International Cooperation and Exchange Project (61120106009) of National Natural Science Foundation of China, NFS grant ECCS1405173, ONR grant N00014-13-1-0562, ONR grant N000141410718, and China Education Ministry Project 111 (No.B08015).

E-mail address: jihonghai@gmail.com (H.H. Ji), lewis@uta.edu (F.L. Lewis), zhshhou@bjtu.edu.cn (Z.S. Hou),dmikulski@gmail.com (D. Mikulski).

${ }^{1}$ Tel.:+86 105168 8617; Fax: +86 1051688617 . each agent can only use information from its neighbors in a prescribed communication network. In addition, each agent must reach a consensus estimate that agrees with the estimates of all other nodes in the network. Consensus and synchronization in cooperative multi-agent systems has attracted a great deal of attention since the papers by Jadbabaie et al. (2003) and Olfati-Saber and Murray (2004). In this literature, protocols are sought to ensure synchronization of the states of all the agents to a common value. These protocols must respect the allowed communication links in that they are distributed in the sense that the computations of each agent depend only on the neighbors of that agent in the prescribed communication graph. The motivation for reaching consensus on the states of all agents arises in many fields, and consensus protocols have been applied to generate target velocity and position commands for networked vehicle formation control (Fax \& Murray, 2004), to generate reference frequencies and voltages for 
synchronization in distributed energy generation microgrids (Bidram, Lewis, \& Davoudi, 2014), for attitude synchronization in multiple satellites (Sahoo \& Banavar, 2014), and to explain the synchronization of coupled oscillators (Strogatz \& Stewart, 1993) and the reaching of agreement in human social networks (Wasserman \& Faust, 1994). See the books (Lewis, Zhang, Movric, \& Das, 2014), (Qu, 2009) for comprehensive treatments.

The computation of consensus values in the cooperative control literature has generally not been optimal, nor taken into account channel transmission noises, nor quantified the accuracy of the consensus values reached. As such, the application of distributed Kalman filtering techniques to compute optimal consensus values of the states all agents in a networked team is a natural extension of the current literature.

Decentralized Kalman filtering techniques have attracted much attention and considerable research during the past few decades in sensor networks because they do not need a centralized processing station. The results include faster parallel processing and increased robustness to failures. Early works (Hashemipour, Roy, \& Laub, 1988; Rao \& Durrant-Whyte, 1991; Rao, Durrant-Whyte, \& Sheen, 1993) were applied to data fusion by developing decentralized Kalman filtering algorithms. In Rao and Durrant-Whyte (1991), each node computed its own decentralized local estimate for an unknown target state vector, and a method was given for fusing or assimilating all the local estimates into a single estimate for the target state to accomplish globally optimal performance. Because the information flow is all-to-all with communication complexity of $O\left(N^{2}\right)$ ( $N$ is the number of sensors/nodes), this solution is not scalable for large-scale sensor networks, as stated in Olfati-Saber (2007). Communication complexity of Kalman consensus filtering depends on the information sent at each consensus step between sensors discussed in Kamal et al. (2012). It is evaluated quantitatively by communication bandwidth (BYTES/s) and computation burden (FLOPS/s) in Ferguson and How (2005). In these works, there was generally no requirement for all nodes to reach the same estimate of the target state.

Olfati-Saber (2007), states the difference between the decentralized Kalman filtering and distributed Kalman filtering. It is stated there "the decentralized Kalman filter in Rao, Durrant-Whyte, \& Sheen (1993) involves state estimation using a set of local Kalman filters that communicate with all other nodes. The information flow is all-to-all with communication complexity of $O\left(\mathrm{n}^{2}\right)$ which is not scalable for sensor networks." Here, we focus on scalable or distributed Kalman Filtering algorithms in which each node only communicates messages with its neighbors on a network. Unlike the decentralized Kalman filtering in Rao and Durrant-Whyte (1991), the distributed Kalman filtering is more widely used for multi-sensor fusion and tracking problems recently where nodes need to reach consen- sus estimates at the cost of non-optimal estimates, due to their scalability for large networks and high fault tolerance. A comprehensive review of distributed state estimation approaches and comparisons with centralized and decentralized methods are detailed in Taj and Cavallaro (2011). Besides, the concerns of occlusions or limited observability of sensors in many applications domains, such as a distributed camera network detailed in Kamal et al. (2013), cannot be neglected, which motivates us to develop novel distributed filtering algorithms for data fusion against failure nodes in sensor networks.

The focus of this paper is the distributed Kalman consensus filtering problem, where the objective is to provide a good trade between accuracy and communication cost. The novel distributed Kalman filter could save communication costs in peer-to-peer information flow and improve the network robustness to possible failure of sensors simultaneously. In distributed consensus filtering, a graph topology is imposed that restricts inter-node communications, sensor nodes can directly use only information from local neighbors in the prescribed graph topology, locally optimal estimates are desired for the unknown target state at each node, and it is required that all local estimates eventually reach a consensus agreement about the values of their estimates of the target sensor state.

The work of Spanos et al. (2005a) focuses on dynamic distributed sensor fusion to obtain consensus weighted least-squares fused estimates for multiple measurements. That paper does not include the dynamics of a target and no direct relation is drawn with the optimal Kalman filter. Sophisticated algorithms for diffusion implementation, distributed estimation and inference in sensor networks can be found in, e.g., Spanos et al. (2005b), Spanos et al. (2005c) and Xiao et al. (2006).

The work in Olfati-Saber (2005), Olfati-Saber and Shamma (2005) presented distributed Kalman filtering algorithms, which consist of a network of micro-Kalman filters each embedded with a low-pass and a band-pass consensus filter. The Kalman consensus filter (KCF) proposed in Olfati-Saber (2007) has become a popular and efficient distributed consensus-based framework for dynamic state estimation, where each node sends/receives the measurements to/from only its local neighbors. A formal derivation, stability and performance analysis of KCF was given in Olfati-Saber (2009). The consensus terms in these papers are added in an ad hoc fashion outside the Kalman Filter framework. Moreover, in those works, inaccurate estimates of neighbors caused by limited observability are not discounted in the consensus algorithms. If neither a sensor (node) nor its immediate neighbors can receive messages from the target it is called a Naive sensor node in Kamal et al. (2013), where it was shown that naive nodes can deteriorate the network performance due to their inaccurate estimation values.

Several distributed Kalman filter schemes were given in 
Kamal et al. (2011), Kamal et al. (2013) and Wang et al. (2014) to remedy these issues. In those works, distributed local consensus filters were developed that have information weighting, that is, weighting by the inverse of the estimation error covariance matrix. Simulations showed improved performance relative to that of the KCF (OlfatiSaber, 2007). These works are for the discrete-time case and cannot easily be extended to the continuous-time case.

Another distributed KCF called Multi-agent Kalman consensus filter (MKCF) was given in Ren et al. (2005), Analogous to process and measurement noises, channel communication noises may also deteriorate the accuracy of estimation. To address this issue, both discrete-time and continuous-time MKCF are studied therein. In that work, the resulting filtering algorithms do not contain the state of the target node, so it is not clear that it is actually being estimated, even though a consensus estimate is reached. Alighanbari et al. (2006) proposed a modified version of this algorithm to obtain an unbiased estimation.

The aim of this paper is to develop a novel Informationweighted Kalman Consensus Filter (IKCF), with improved properties, for continuous-time systems. A goal is to provide an optimal distributed consensus filter whose convergence can be formally proven. The approach is to impose the information flow topology up-front by assuming a distributed form for the measurement matrices of the sensor nodes. Then, the Kalman filter equations are formally applied to develop locally optimal distributed consensus filters wherein the effects of poor estimates of neighbors are discounted using information weighting terms.

The contributions of the IKCF in this paper are as follows.

1) The information flow topology is formally captured upfront in the Kalman filter formulation by defining a distributed information-weighted measurement model for each node. Both process noises and channel communication noises are included.

2) Locally optimal estimates are then derived based on the distributed measurement model at each node by formally employing the Kalman Filter equations. The result is a fully distributed Kalman consensus filter that includes information weighting to discount faulty estimates from neighbor nodes.

3) A formal proof of convergence of the distributed IKCF is given using Lyapunov techniques.

4) The distributed IKCF is further extended to account for state estimation of a target state that is driven by an unknown input. A formal convergence analysis is again provided.

5) Simulations show the improved performance of the IKCF in this paper relative to the KCFs in other papers.

The paper is organized as follows. Section 2 introduces background about graph theory and defines the distributed consensus filtering problem. Section 3 reviews existing background results for Kalman consensus filters on graphs.
In Section 4, the new distributed IKCF is developed for sensor networks. Moreover, the convergence of IKCF is formally proven by using Lyapunov stability theory. The proposed distributed IKCF is additionally extended for the state estimation problem when the target node dynamics is driven by an unknown input in Section 5. Section 6 presents simulation results and shows that the new IKCF outperforms some existing distributed filtering algorithms.

\section{Background and formulation of distributed consen- sus filtering problem}

This section introduces some basics of graph theory and the standard continuous-time Kalman filter (CTKF). Then the distributed consensus filtering problem is stated.

\subsection{Graph theory in sensor networks}

Standard graph theory notions are used in Lewis et al. (2014). For a sensor network, a collection of $N$ sensor nodes are connected with each other using the directed graph interaction topology $G=(\mathcal{V}, \mathcal{E}, \mathcal{A})$, where $\mathcal{V}=\{1,2, \ldots, N\}$ is the set of vertices representing the sensors, $\mathcal{E} \subseteq \mathcal{V} \times \mathcal{V}$ is the set of edges that stand for the communication channels, and $\mathcal{A}=\left[a_{i j}\right] \in \mathfrak{R}^{N \times N}$ is the associated weighted adjacency matrix. Define the in-degree of sensor $i$ as $d_{i}=\sum_{j=1}^{N} a_{i j}$ and in-degree matrix as $D=\operatorname{diag}\left\{d_{i}\right\} \in \mathfrak{R}^{N \times N}$. Then the graph Laplacian matrix is $L=D-\mathcal{A}$. An edge $(j, i) \in \mathcal{E}$, graphically denoted by an arrow with head node $i$ and tail node $j$, implies an open communication channel from sensor $j$ to sensor $i$. Each entry $a_{i j}$ of adjacency matrix is the weight associated with edge $(j, i)$ and $a_{i j}=1$ if $(j, i) \in \mathcal{E}$. Otherwise, $a_{i j}=0$. A directed path from sensor $i$ to sensor $j$ is a sequence of successive edges in the form $\{(i, k),(k, l), \ldots(m, j)\}$. A (directed) tree is a connected digraph where every node except one, called the root node, has in-degree equal to one. A spanning tree of a digraph is a directed tree, for some root node, formed by graph edges that connects all the nodes of the graph. A graph is said to have a spanning tree, for some root node, if a subset of the edges forms a directed tree. The set of neighbors of sensor $i$ is denoted by $\mathcal{N}_{i}=\{j \mid(j, i) \in \mathcal{E}, \forall j \neq i\}$. Symbol $\otimes$ represents the Kronecker product.

\subsection{The standard continuous-time Kalman filter}

The standard CTKF is given as follows (Lewis, Xie, \& Popa, 2008; Crassidis \& Junkins, 2004). For notational convenience, the time arguments are sometimes omitted when no confusion arises throughout this paper. Consider the CT linear time invariant system 


$$
\dot{x}=A x+B u+F w
$$

where $x \in \mathfrak{R}^{n}, x(0) \sim\left(\bar{x}_{0}, P_{0}\right)$ and the process noise is $w \sim(0, S)$. Consider the measurements

$$
z=H x+v
$$

where the measurement noise is $v \sim(0, R)$. Assume $\{w(t)\}$ and $\{v(t)\}$ are zero-mean white Gaussian noise (WGN) uncorrelated with each other and with $x(0)$, and that the pair $(A, H)$ is observable. The CTKF equations are given by

$$
\begin{aligned}
& \dot{P}=A P+P A^{T}+F S F^{T}-P H^{T} R^{-1} H P, \\
& K=P H^{T} R^{-1}, \\
& \dot{\hat{x}}=A \hat{x}+B u+K(z-H \hat{x})
\end{aligned}
$$

where the state estimation error covariance matrices are $P=E\left\{(\hat{x}-x)(\hat{x}-x)^{T}\right\} \in \mathfrak{R}^{n \times n}$. This standard CTKF gives estimates $\hat{x}(t)$ of $x(t)$ that are optimal in a least-squares sense.

\subsection{Distributed consensus filtering problem}

In this paper, the state $x(t)$ to be estimated is considered as the "target node". The focus of this paper is the problem defined as follows.

Distributed Consensus Filtering Problem. Given a sensor graph, the objective of each node is to estimate the state of a target or leader node, denoted as node 0 , which is endowed with dynamics (1). Locally optimal estimates are desired for the unknown state at each node. In addition, all nodes should eventually reach a consensus agreement about the values of their estimates of the state of node 0 .

In the following sections it is shown how to use the Kalman filter equations to develop a novel distributed Kalman consensus filter (KCF) scheme that can be implemented at each node using only locally available information.

The following assumptions are needed.

Assumption 1. The graph has a spanning tree and the target node can be observed by at least one root node. That is $g_{i 0} \neq 0$ for at least one root node $i$.

Assumption 2. The target's state $x(t)$ is collectively observable by all the sensors, i.e., the pair $(A, H)$ with $H=\left(H_{1}^{T}, \ldots, H_{N}^{T}\right)^{T}$ is observable.

\section{Background results for Kalman consensus filters on graphs}

The objective of this section is to briefly review the background for Kalman consensus filtering given in previous work (Spanos et al., 2005a; Olfati-Saber, 2007;
Kamal et al., 2011; Kamal et al., 2013; Ren et al., 2005) and explain the motivation for the work in this paper.

\subsection{Weighted least-squares distributed consensus filter}

In Spanos et al. (2005a) the following consensus filter is given. Define the weighting matrices $W_{i}(t)$. The following update equations for the estimates at each node are used.

$$
\begin{aligned}
& \dot{\hat{x}}_{i}^{-}=W_{i} \dot{z}_{i}+\dot{W}_{i} z_{i}+\sum_{j \in N_{i}}\left(\hat{x}_{j}^{-}-\hat{x}_{i}^{-}\right), \\
& \dot{M}_{i}=\dot{W}_{i}+\sum_{j \in N_{i}}\left(M_{j}-M_{i}\right), \\
& \hat{x}_{i}=M_{i}^{-1} \hat{x}_{i}^{-}
\end{aligned}
$$

with the initial condition $\hat{x}_{i}^{-}(0)=W_{i}(0) z_{i}(0)$ and $M_{i}(0)=W_{i}(0)$. Define $P_{i}=M_{i}^{-1}$, then equations (6)-(8) can be written as

$$
\begin{aligned}
\dot{P}_{i} & =-P_{i} \dot{W}_{i} P_{i}-P_{i} \sum_{j \in N_{i}}\left(P_{j}^{-1}-P_{i}^{-1}\right) P_{i}, \\
\dot{\hat{x}}_{i} & =P_{i} W_{i} \dot{z}_{i}+P_{i} \dot{W}_{i}\left(z_{i}-\hat{x}_{i}^{-}\right)+P_{i} \sum_{j \in N_{i}}\left(\hat{x}_{j}^{-}-P_{j}^{-1} P_{i} \hat{x}_{i}^{-}\right) \\
& =P_{i} W_{i} \dot{z}_{i}+P_{i} \dot{W}_{i}\left(z_{i}-P_{i}^{-1} \hat{x}_{i}\right)+P_{i} \sum_{j \in N_{i}} P_{j}^{-1}\left(\hat{x}_{j}-\hat{x}_{i}\right) .
\end{aligned}
$$

It is confirmed in Spanos et al. (2005a) that the outputs $\hat{x}_{i}$ all track a consensus given by a weighted average of the $z_{i}(t)$ with zero steady-state error. The final consensus value is the instantaneous least-squares estimate $\lim _{t \rightarrow \infty} \hat{x}_{i}=\left(\sum_{j \in N_{i}} W_{i}(\infty)\right)^{-1}\left(W_{i}(\infty) z_{i}(\infty)\right)$.

In Spanos et al. (2005a), dynamics of a target such as (1) are not included. The dynamical information is obtained through measurements of velocities $\dot{z}_{i}(t)$. Also, each node has a measurement model such as (2) with $H_{i}=I$ and noise covariances $R_{i}$. This results in the relation of weighting matrices to noise covariances given by $W_{i}=R_{i}^{-1}$. The requirement that $H_{i}=I, \forall i$ is very restrictive.

\subsection{Distributed Kalman consensus filtering for sensor networks}

The scheme proposed in (Olfati-Saber, 2007) makes a simple modification of the CTKF equations (3)-(5) to introduce a consensus term as follows.

The dynamics of the target node are (1), with input $u(t)$ equal to zero, and each node has measurements available given by

$$
z_{i}=H_{i} x+v_{i}
$$

where the measurement noise is $v_{i} \sim\left(0, R_{i}\right)$. Assumption 2 is made.

Each node runs a local Kalman filter according to the equations 


$$
\begin{aligned}
& \dot{P}_{i}=A P_{i}+P_{i} A^{T}+F S F^{T}-P_{i} H_{i}^{T} R_{i}^{-1} H_{i} P_{i}, \\
& K_{i}=P_{i} H_{i}^{T} R_{i}^{-1}, \\
& \dot{\hat{x}}_{i}=A \hat{x}_{i}+K_{i}\left(z_{i}-H_{i} \hat{x}_{i}\right)+\gamma P_{i} \sum_{j \in N_{i}}\left(\hat{x}_{j}-\hat{x}_{i}\right)
\end{aligned}
$$

where $\gamma>0$ and the distributed state estimation error covariance matrices are $P_{i}=E\left\{\left(\hat{x}_{i}-x\right)\left(\hat{x}_{i}-x\right)^{T}\right\}$. The last consensus term in (14) is added and it takes into account the state estimates of the neighbors of node $i$.

It is proven in Olfati-Saber (2007) that, if there is no process or measurement noise, then all nodes reach consensus estimates such that $\hat{x}_{i} \rightarrow x$.

Some deficiencies have been noted in this approach. First, the consensus term in (14) is introduced in an ad hoc manner outside the optimal Kalman filter framework. As such, the resultant estimates can no longer be guaranteed to be locally optimal in a least-squares sense, especially in the presence of communication noise between sensors. Second, the estimates of neighbor nodes $\hat{x}_{j}$ in (14) are not discounted if their error covariances $P_{j}$ are large. By contrast the consensus terms $\left(\hat{x}_{j}-\hat{x}_{i}\right)$ from agent $j$ are weighted by $P_{j}^{-1}$ in (10). If neither a sensor (node) nor its immediate neighbors can measure the target node output, it is has been called in the literature a "naive node". The presence of naive nodes has been shown to deteriorate the performance of the distributed consensus filter (12)-(14) in Kamal et al. (2013).

\subsection{Multi-agent Kalman consensus with relative uncer- tainly}

The work of Ren et al. (2005) considers the case of a static target node, that is $A=0, B=0, F=I$ in (1). Given the following measurement model for each node

$$
z_{i}=H_{i} x+v_{i}=\left(\begin{array}{c}
a_{i 1}\left(\hat{x}_{1}+\omega_{i 1}\right) \\
\vdots \\
a_{i N}\left(\hat{x}_{N}+\omega_{i N}\right)
\end{array}\right)
$$

where $\omega_{i j}$ are the channel communication (or transmission) noises, it develops the Multi-agent Kalman consensus filter (MKCF) given by

$$
\begin{aligned}
& \dot{P}_{i}=-P_{i}\left[\sum_{j=1}^{N} a_{i j}\left(P_{j}+\Pi_{i j}\right)^{-1}\right] P_{i}+S, \\
& K_{i j}=P_{i}\left(P_{j}+\Pi_{i j}\right)^{-1} \\
& \dot{\hat{x}}_{i}=\sum_{j=1}^{N} a_{i j} K_{i j}\left(\left(\hat{x}_{j}+\omega_{i j}\right)-\hat{x}_{i}\right) .
\end{aligned}
$$

where $\Pi_{i j}=E\left\{\omega_{i j} \omega_{i j}^{T}\right\}$ for all $i=1,2, \ldots, N, j \in \mathcal{N}_{i}$.

Note that neither the measurement model (15) nor the MKCF equations (16)-(18) contain the target's state $x(t)$, so it is not clear what sort of consensus estimate is reached.
That is, no agent has any direct measurements of the target's dynamics. Moreover, the proof in Ren et al. (2005) assumes scalar Kalman gains $K_{i j}$ that are positive, whereas in fact $K_{i j}$ are matrices and may not be positive definite.

The next result corrects the first deficiency and extends the work of Ren et al. (2005) to the general target dynamics (1).

Lemma 1. Consider the target dynamics (1) with the measurement model

$$
z_{i}=\left(\begin{array}{c}
g_{i 0}\left(x+v_{i}\right) \\
a_{i 1}\left(\hat{x}_{1}+\omega_{i 1}\right) \\
\vdots \\
a_{i N}\left(\hat{x}_{N}+\omega_{i N}\right)
\end{array}\right)
$$

where $v_{i}$ is the process noise. Then one has the distributed $\mathrm{MKCF}$ given by

$$
\begin{aligned}
\dot{P}_{i} & =A P_{i}+P_{i} A^{T}+F S F^{T} \\
& -P_{i}\left[\sum_{j=1}^{N} a_{i j}\left(P_{j}+\Pi_{i j}\right)^{-1}+g_{i 0} \Omega_{i}^{-1}\right] P_{i}, \\
\dot{\hat{x}}_{i} & =A \hat{x}_{i}+B u+g_{i 0} P_{i} \Omega_{i}^{-1}\left(x-\hat{x}_{i}\right) \\
& +P_{i} \sum_{j=1}^{N} a_{i j}\left(P_{j}+\Pi_{i j}\right)^{-1}\left(\left(\hat{x}_{j}+v_{i j}\right)-\hat{x}_{i}\right) .
\end{aligned}
$$

where $\Omega_{i}=E\left\{v_{i} v_{i}^{T}\right\}$.

Proof. Similar to the proof of Theorem 1 below.

Comparing the structure (19) to (15), it is seen that available are direct measurements of the target node in (19) if sensor (node) $i$ can obtain direct information from the target, that is when $g_{i 0}=1$. Only a few nodes $i$ need have direct access to the target's state measurements, as seen in Section 4.2 below.

Although this information model (19) is highly interesting, the proof of convergence of (20), (21) is elusive so far. It is still an open issue. Our own work is in fact motivated by these results extended from Ren et al. (2005), but we use a different structure in the next section that admits a convergence proof.

\section{Optimal distributed information-weighted Kalman consensus filter (IKCF)}

In this section, a novel distributed Kalman consensus filter $(\mathrm{KCF})$ is developed. It is desired to obtain locally optimal sensor fusion algorithms. Therefore, this scheme is derived by considering the dynamics (1) and applying the Kalman filter (3)-(5) assuming from the beginning specific information measurement structures in (11) that depend on the imposed communication topology. We assume that the target dynamics $A, B$ and $F$ in (1) and the adjacency matrix $\mathcal{A}=\left[a_{i j}\right]$ are both known to all sensors in the network. Also, a specific form of the measurement matrices 
$H_{i}$ is selected up-front that depends on locally available information at each agent. This scheme gives locally optimal least-squares estimates using distributed computation filtering protocols. The resulting local consensus filters include information weighting such as $P_{j}^{-1}$ in (10). Moreover, these filters provide improved performance over the existing schemes, as shown by rigorous convergence analysis and guarantees of optimality in this section, and simulations in Section 6.

\subsection{Derivation of distributed information-weighted Kal- man consensus filter}

Here, a novel distributed Kalman consensus filter is developed, wherein the estimates from neighbor nodes $j$ are weighted by their information content and the estimates of all nodes converge to the optimal estimate for a target node. We call this the distributed information-weighted Kalman consensus filter (IKCF). In this distributed IKCF, a structure is selected up-front for $H_{i}$ in (11) that depends on graph communication topology, and the Kalman filter equations (3)-(5) are then applied at each node. The result is a novel distributed IKCF that is locally optimal.

The information flow topology is formally captured in the Kalman filter formulation by defining the distributed measurement model of the $i$-th sensor in the form

$$
z_{i}=\left(\begin{array}{c}
g_{i 0}\left(\bar{H}_{i} x+v_{i}\right) \\
\sum_{j=1}^{N} a_{i j} P_{j}^{-1}\left(\hat{x}_{j}+\omega_{i j}\right)
\end{array}\right) \equiv H_{i}(t) x(t)+v_{i}(t)
$$

where $\bar{H}_{i}$ are distributed observation matrices, error covariance is $P_{i}=E\left\{\left(\hat{x}_{i}-x\right)\left(\hat{x}_{i}-x\right)^{T}\right\} \quad, \quad v_{i} \quad$ is $\quad$ a measurement noise, and channel transmission (or communication) noises are $\omega_{i j}(t)$.

This novel information flow topology captured in measurement model (22) is an extension form of the standard model (11) that consists of direct and indirect target state measurements, which can be written in the standard form (11) with

$$
H_{i}=\left(\begin{array}{c}
g_{i 0} \bar{H}_{i} \\
\sum_{j=1}^{N} a_{i j} P_{j}^{-1}
\end{array}\right), \quad v_{i}=\left(\begin{array}{c}
g_{i 0} v_{i} \\
\sum_{j=1}^{N} a_{i j} P_{j}^{-1}\left(\hat{x}_{j}-x+\omega_{i j}\right)
\end{array}\right) .
$$

It is seen in Section 4.2 that the new distributed IKCF model (22) allows for a formal proof of convergence.

This measurement model only uses information about the estimates of the neighbors $j \in \mathcal{N}_{i}$ of node $i$. If sensor (node) $i$ can obtain direct information from the target, then $g_{i 0}=1$ and $\bar{H}_{i} \neq 0$, otherwise $g_{i 0}=0$ and $\bar{H}_{i}=0$. It is required that at least one root node have direct information from the target. Several nodes may have direct measurements of the target, and measurement matrices $\bar{H}_{i}$ may be different if $g_{i 0} \neq 0$ for more than one node $i$.
Note that $P_{j}^{-1}$ is the Fisher information matrix (Lewis, Xie, \& Popa, 2008) of node $j$, which is larger if the estimates of node $j$ are more precise. The information weighting in (22) is motivated by (10) and by recent discoveries in Kamal et al. (2011), Kamal et al. (2013) and Wang et al. (2014).

We call the nodes for which $g_{i 0} \neq 0$ "informed nodes" because they have direct access to output meaurements $\bar{H}_{i} x$ of the target state. Measurement matrices $\bar{H}_{i}$ can generally be different. If neither a sensor (node) nor its immediate neighbors can measure the target node output, it is has been called in the literature a "naive node". In our formulation, however, this nomenclature is not required since naive nodes do not deteriorate the consensus estimates., as proven in Theorem 2.

Remark 1. Note that several informed root nodes may have direct access to output measurements of the target state. As such, the problem is how to combine these several measurements by informed nodes into a single consensus optimal estimate of $x(t)$. The informed nodes may be across the network from each other- as such the estimates can only be optimally combined by propagation of information through the communication graph. Note that this may require that the so-called "naive nodes" participate in the information exchange process to obtain the optimal consensus estimates.

The following main result develops the distributed IKCF based on the distributed measurement model (22).

Theorem 1: Distributed IKCF. Given the target's state equation (1) and the distributed information flow structure (22), the standard CTKF equations (3)-(5) can be written as the distributed IKCF with the covariance propagation equations

$$
\begin{aligned}
\dot{P}_{i} & =A_{i} P_{i}+P_{i} A_{i}^{T}+F S F^{T}-g_{i 0}^{2} P_{i} \bar{H}_{i}^{T} \Omega_{i}^{-1} \bar{H}_{i} P_{i} \\
& -P_{i}\left[\left(\sum_{j=1}^{N} a_{i j} P_{j}^{-1}\right)\left(\sum_{j=1}^{N} a_{i j}\left(P_{j}^{-1}+P_{j}^{-1} \Pi_{i j} P_{j}^{-1}\right)\right)^{-1}\right. \\
& \left.\cdot\left(\sum_{j=1}^{N} a_{i j} P_{j}^{-1}\right)-\sum_{j=1}^{N} a_{i j} P_{i}^{-1}\right] P_{i}
\end{aligned}
$$

where $A_{i}=A+\sum_{j=1}^{N} a_{i j} I_{n}, \Omega_{i}=E\left\{g_{i 0}^{2} v_{i} v_{i}^{T}\right\}, \Pi_{i j}=E\left\{\omega_{i j} \omega_{i j}^{T}\right\}$ $, i, j=1,2, \ldots N$. Define the direct Kalman gain associated with direct target measurements and the indirect Kalman gain associated with neighbors' estimates respectively as

$$
\begin{aligned}
& K_{i}^{\text {dir }}=g_{i 0} P_{i} \bar{H}_{i}^{T} \Omega_{i}^{-1}, \\
& K_{i}^{\text {ind }}=P_{i}\left(\sum_{j=1}^{N} a_{i j} P_{j}^{-1}\right)\left(\sum_{j=1}^{N} a_{i j}\left(P_{j}^{-1}+P_{j}^{-1} \Pi_{i j} P_{j}^{-1}\right)\right)^{-1} .
\end{aligned}
$$

Then, the distributed agent estimate updates are given as 


$$
\begin{aligned}
\dot{\hat{x}}_{i} & =A \hat{x}_{i}+B u+g_{i 0} K_{i}^{\text {dir }}\left(\bar{H}_{i}\left(x-\hat{x}_{i}\right)+v_{i}\right) \\
& +K_{i}^{i n d} \sum_{j=1}^{N} a_{i j} P_{j}^{-1}\left(\hat{x}_{j}-\hat{x}_{i}+\omega_{i j}\right) .
\end{aligned}
$$

Proof. Write the measurement model (22) equivalently as follows. Define the (direct) term consisting of direct target state measurements as

$$
z_{i}^{d i r}=g_{i 0}\left(\bar{H}_{i} x+v_{i}\right)=H_{i}^{d i r} x+v_{i}^{d i r}
$$

where $H_{i}^{d i r}=g_{i 0} \bar{H}_{i}$ denotes the direct measured information flow structure, $v_{i}^{\text {dir }}=g_{i 0} v_{i}$ is the direct measurement noise for all $i=1,2, \ldots N$. Define the (indirect) term depending on the estimates of the neighbors of node $i$ as

$$
\begin{aligned}
z_{i}^{i n d} & =\sum_{j=1}^{N} a_{i j} P_{j}^{-1}\left(\hat{x}_{j}+\omega_{i j}\right)=H_{i}^{i n d} x+v_{i}^{i n d} \\
& =\sum_{j=1}^{N} a_{i j} P_{j}^{-1} x+\sum_{j=1}^{N} a_{i j} P_{j}^{-1}\left(\hat{x}_{j}-x+\omega_{i j}\right) .
\end{aligned}
$$

Here, $H_{i}^{\text {ind }}=\sum_{j=1}^{N} a_{i j} P_{j}^{-1}$ can be considered as the indirect measured information flow structure and $v_{i}^{i n d}=\sum_{j=1}^{N} a_{i j} P_{j}^{-1}\left(\hat{x}_{j}-x+\omega_{i j}\right)$ as the indirect measurement noise.

Consider the target's dynamics (1) with estimation error covariance weighted measurement model (28) and (29), the distributed Kalman filter structure for state estimation is given by

$$
\begin{aligned}
\dot{\hat{x}}_{i}= & A \hat{x}_{i}+B u+K_{i}\left(z_{i}-H_{i} \hat{x}_{i}\right) \\
= & A \hat{x}_{i}+B u+K_{i}^{d i r}\left(z_{i}^{d i r}-H_{i}^{d i r} \hat{x}_{i}\right)+K_{i}^{\text {ind }}\left(z_{i}^{i n d}-H_{i}^{i n d} \hat{x}_{i}\right) \\
= & A \hat{x}_{i}+B u+g_{i 0} K_{i}^{d i r}\left(\bar{H}_{i}\left(x-\hat{x}_{i}\right)+v_{i}\right) \\
& +K_{i}^{i n d} \sum_{j=1}^{N} a_{i j} P_{j}^{-1}\left(\hat{x}_{j}-\hat{x}_{i}+\omega_{i j}\right) \\
= & \left(A+\sum_{j=1}^{N} a_{i j} I_{n}\right) \hat{x}_{i}+g_{i 0} K_{i}^{d i r}\left(\bar{H}_{i}\left(x-\hat{x}_{i}\right)+v_{i}\right) \\
& +K_{i}^{i n d} \sum_{j=1}^{N} a_{i j} P_{j}^{-1}\left(\hat{x}_{j}-\hat{x}_{i}+\omega_{i j}\right)-\sum_{j=1}^{N} a_{i j} \hat{x}_{i}+B u
\end{aligned}
$$

where $K_{i}=\left(\begin{array}{ll}K_{i}^{d i r} & K_{i}^{\text {ind }}\end{array}\right)$ and $K_{i}^{\text {dir }}$ and $K_{i}^{\text {ind }}$ are Kalman gains for direct and indirect measurements.

Rewrite the (30) as follows

$$
\begin{aligned}
\dot{\hat{x}}_{i} & =A_{i} \hat{x}_{i}+B u+g_{i 0} K_{i}^{d i r}\left(\bar{H}_{i}\left(x-\hat{x}_{i}\right)+v_{i}\right) \\
& +K_{i}^{i n d} \sum_{j=1}^{N} a_{i j} P_{j}^{-1}\left(\hat{x}_{j}-\hat{x}_{i}+\omega_{i j}\right)-\sum_{j=1}^{N} a_{i j} \hat{x}_{i} .
\end{aligned}
$$

Define the distributed state synchronization error dynamic as $\tilde{x}_{i}=x-\hat{x}_{i}$, the distributed state error dynamics are derived from (1) and (31) as

$$
\begin{aligned}
\dot{\tilde{x}}_{i} & =A x+B u-A_{i} \hat{x}_{i}-B u-g_{i 0} K_{i}^{d i r}\left(\bar{H}_{i}\left(x-\hat{x}_{i}\right)+v_{i}\right) \\
& -K_{i}^{i n d} \sum_{j=1}^{N} a_{i j} P_{j}^{-1}\left(\hat{x}_{j}-\hat{x}_{i}+\omega_{i j}\right)+\sum_{j=1}^{N} a_{i j} \hat{x}_{i}+F w \\
= & A_{i} \tilde{x}_{i}-g_{i 0} K_{i}^{d i r} \bar{H}_{i} \tilde{x}_{i}+F w-g_{i 0} K_{i}^{d i r} v_{i} \\
& +K_{i}^{i n d} \sum_{j=1}^{N} a_{i j} P_{j}^{-1}\left(\tilde{x}_{j}-\tilde{x}_{i}+\omega_{i j}\right)-\sum_{j=1}^{N} a_{i j} \tilde{x}_{i} .
\end{aligned}
$$

Furthermore, (32) becomes

$$
\dot{\tilde{x}}_{i}=E_{i} \tilde{x}_{i}+T_{i}
$$

where

$$
E_{i}=A_{i}-g_{i 0} K_{i}^{d i r} \bar{H}_{i}-K_{i}^{\text {ind }} \sum_{j=1}^{N} a_{i j} P_{j}^{-1}
$$

and

$$
\begin{aligned}
T_{i} & =F w-g_{i 0} K_{i}^{d i r} v_{i}+K_{i}^{i n d} \sum_{j=1}^{N} a_{i j} P_{j}^{-1}\left(\tilde{x}_{j}+\omega_{i j}\right)-\sum_{j=1}^{N} a_{i j} \tilde{x}_{i} \\
& =F w-g_{i 0} K_{i}^{d i r} v_{i}+K_{i}^{i n d} v_{i}-d_{i} \tilde{x}_{i} .
\end{aligned}
$$

In (35), $v_{i}=\sum_{j=1}^{N} a_{i j} P_{j}^{-1}\left(\tilde{x}_{j}+\omega_{i j}\right)$ for all $i=1,2, \ldots N$ and are zero-mean Gaussian state-dependent noise processes. Assume that processes noise $w(t)$ of the target's dynamics (1), the observation noises $v_{i}(t)$ of different sensors, and the communication channel noises $\omega_{i j}(t)$ are mutually uncorrelated. Then, from (33), is follows that the $w, v_{i}$ and $v_{i}$ are uncorrelated since $v_{i}$ directly depends on $\tilde{x}_{i}$ and not $w$ or $v_{i}$. Therefore, all the expectations of cross terms are equal to zeroes.

Define noise covariance matrices as

$$
\begin{aligned}
& S=E\left\{w w^{T}\right\}, \\
& R_{i}^{d i r}=E\left\{g_{i 0}^{2} v_{i} v_{i}^{T}\right\}=\Omega_{i}, \\
& R_{i}^{\text {ind }}=E\left\{v_{i} v_{i}^{T}\right\}=\sum_{j=1}^{N} a_{i j}\left(P_{j}^{-1}+P_{j}^{-1} \Pi_{i j} P_{j}^{-1}\right) . \\
& \text { Then } \\
& \begin{aligned}
E\left\{T_{i}(t) T_{i}^{T}(\tau)\right\} & =\left(F S F^{T}+K_{i}^{d i r} R_{i}^{\text {dir }}\left(K_{i}^{\text {dir }}\right)^{T}\right. \\
& \left.+K_{i}^{\text {ind }} R_{i}^{\text {ind }}\left(K_{i}^{\text {ind }}\right)^{T}+d_{i} P_{i}\right) \delta(t-\tau) .
\end{aligned}
\end{aligned}
$$

Using the matrix exponential solution gives

$$
\tilde{x}_{i}(t)=\Phi_{i}\left(t, t_{0}\right) \tilde{x}_{i}\left(t_{0}\right)+\int_{t_{0}}^{t} \Phi_{i}(t, \tau) T_{i} d \tau
$$

Substituting equation (40) into equation $P_{i}=E\left\{\left(\hat{x}_{i}-x\right)\left(\hat{x}_{i}-x\right)^{T}\right\}$ with Assumption 1, leads to

$$
\begin{aligned}
P_{i}(t) & =\Phi_{i}\left(t, t_{0}\right) P_{i}\left(t_{0}\right) \Phi_{i}^{T}\left(t, t_{0}\right) \\
& +\int_{t_{0}}^{t} \Phi_{i}(t, \tau)\left[F S F^{T}+K_{i}^{\text {dir }} R_{i}^{d i r}\left(K_{i}^{d i r}\right)^{T}\right. \\
& \left.+K_{i}^{\text {ind }} R_{i}^{\text {ind }}\left(K_{i}^{\text {ind }}\right)^{T}+d_{i} P_{i}\right] \Phi_{i}^{T}(t, \tau) d \tau .
\end{aligned}
$$

Taking the time derivative of equation (41) and using the properties of the matrix exponential leads to 


$$
\begin{aligned}
\dot{P}_{i}(t) & =E_{i}(t) \Phi_{i}\left(t, t_{0}\right) P\left(t_{0}\right) \Phi_{i}^{T}\left(t, t_{0}\right) \\
& +\Phi_{i}\left(t, t_{0}\right) P\left(t_{0}\right) \Phi_{i}^{T}\left(t, t_{0}\right) E_{i}^{T}(t)+F S F^{T} \\
& +E_{i}(t) \int_{t_{0}}^{t} \Phi_{i}(t, \tau)\left[F S F^{T}+K_{i}^{\text {dir }} R_{i}^{d i r}\left(K_{i}^{d i r}\right)^{T}\right. \\
& \left.+K_{i}^{\text {ind }} R_{i}^{\text {ind }}\left(K_{i}^{\text {ind }}\right)^{T}+d_{i} P_{i}\right] \Phi_{i}^{T}(t, \tau) d \tau \\
& +\int_{t_{0}}^{t} \Phi_{i}(t, \tau)\left[F S F^{T}+K_{i}^{\text {dir }} R_{i}^{\text {dir }}\left(K_{i}^{\text {dir }}\right)^{T}\right. \\
& \left.+K_{i}^{\text {ind }} R_{i}^{\text {ind }}\left(K_{i}^{\text {ind }}\right)^{T}+d_{i} P_{i}\right] \Phi_{i}^{T}(t, \tau) d \tau E_{i}^{T}(t) \\
& +K_{i}^{\text {dir }} R_{i}^{\text {dir }}\left(K_{i}^{\text {dir }}\right)^{T}+K_{i}^{\text {ind }} R_{i}^{\text {ind }}\left(K_{i}^{\text {ind }}\right)^{T}+d_{i} P_{i} .
\end{aligned}
$$

Using equations (34) and (41) in equation (42) simplifies the expression for $\dot{P}_{i}(t)$ significantly to

$$
\begin{aligned}
\dot{P}_{i} & =\left(A_{i}-g_{i 0} K_{i}^{d i r} \bar{H}_{i}-K_{i}^{\text {ind }} \sum_{j=1}^{N} a_{i j} P_{j}^{-1}\right) P_{i} \\
& +P_{i}\left(A_{i}-g_{i 0} K_{i}^{\text {dir }} \bar{H}_{i}-K_{i}^{\text {ind }} \sum_{j=1}^{N} a_{i j} P_{j}^{-1}\right)^{T} \\
& +F S F^{T}+K_{i}^{\text {dir }} R_{i}^{\text {dir }}\left(K_{i}^{\text {dir }}\right)^{T}+K_{i}^{\text {ind }} R_{i}^{\text {ind }}\left(K_{i}^{\text {ind }}\right)^{T}+d_{i} P_{i} .
\end{aligned}
$$

In order to determine the gains $K_{i}^{\text {dir }}$ and $K_{i}^{\text {ind }}$, minimize the trace of $\dot{P}_{i}$

$$
\begin{aligned}
& \min \quad J\left(K_{i}^{\text {dir }}\right)=\operatorname{Tr}\left(\dot{P}_{i}\right), \\
& \min \quad J\left(K_{i}^{\text {ind }}\right)=\operatorname{Tr}\left(\dot{P}_{i}\right) .
\end{aligned}
$$

Then necessary conditions lead to

$$
\begin{aligned}
& 0=\frac{\partial J}{\partial K_{i}^{d i r}}=2 K_{i}^{\text {dir }} R_{i}^{d i r}-2 g_{i 0} P_{i} \bar{H}_{i}^{T}, \\
& 0=\frac{\partial J}{\partial K_{i}^{\text {ind }}}=2 K_{i}^{\text {ind }} R_{i}^{\text {ind }}-2 P_{i} \sum_{j=1}^{N} a_{i j} P_{j}^{-1} .
\end{aligned}
$$

Solving equations (46) and (47) for $K_{i}^{d i r}$ and $K_{i}^{\text {ind }}$ gives the Kalman gains (25) and (26) as

$$
\begin{aligned}
& K_{i}^{d i r}=g_{i 0} P_{i} \bar{H}_{i}^{T}\left(R_{i}^{d i r}\right)^{-1}=g_{i 0} P_{i} \bar{H}_{i}^{T} \Omega_{i}^{-1}, \\
& K_{i}^{\text {ind }}=P_{i}\left(\sum_{j=1}^{N} a_{i j} P_{j}^{-1}\right)\left(R_{i}^{i n d}\right)^{-1} \\
& =P_{i}\left(\sum_{j=1}^{N} a_{i j} P_{j}^{-1}\right)\left(\sum_{j=1}^{N} a_{i j}\left(P_{j}^{-1}+P_{j}^{-1} \Pi_{i j} P_{j}^{-1}\right)\right)^{-1},
\end{aligned}
$$

The distributed error covariance update (24) is derived from (43) as

$$
\begin{aligned}
& \dot{P}_{i}=A_{i} P_{i}+P_{i} A_{i}^{T}+F S F^{T}+d_{i} P_{i}-g_{i 0}^{2} P_{i} \bar{H}_{i}^{T}\left(R_{i}^{d i r}\right)^{-1} \bar{H}_{i} P_{i} \\
& -P_{i}\left(\sum_{j=1}^{N} a_{i j} P_{j}^{-1}\right)\left(R_{i}^{i n d}\right)^{-1}\left(\sum_{j=1}^{N} a_{i j} P_{j}^{-1}\right) P_{i} \\
& =A_{i} P_{i}+P_{i} A_{i}^{T}+F S F^{T}+d_{i} P_{i}-g_{i 0}^{2} P_{i} \bar{H}_{i}^{T} \Omega_{i}^{-1} \bar{H}_{i} P_{i}-P_{i} \\
& \cdot\left(\sum_{j=1}^{N} a_{i j} P_{j}^{-1}\right)\left(\sum_{j=1}^{N} a_{i j}\left(P_{j}^{-1}+P_{j}^{-1} \Pi_{i j} P_{j}^{-1}\right)\right)^{-1}\left(\sum_{j=1}^{N} a_{i j} P_{j}^{-1}\right) P_{i} .
\end{aligned}
$$

Rearranging the terms of (50) leads to the following update of covariance matrix $P_{i}$

$$
\begin{aligned}
\dot{P}_{i} & =A_{i} P_{i}+P_{i} A_{i}^{T}+F S F^{T}-g_{i 0}^{2} P_{i} \bar{H}_{i}^{T} \Omega_{i}^{-1} \bar{H}_{i} P_{i} \\
& -P_{i}\left[\left(\sum_{j=1}^{N} a_{i j} P_{j}^{-1}\right)\left(\sum_{j=1}^{N} a_{i j}\left(P_{j}^{-1}+P_{j}^{-1} \Pi_{i j} P_{j}^{-1}\right)\right)^{-1}\right. \\
& \left.\cdot\left(\sum_{j=1}^{N} a_{i j} P_{j}^{-1}\right)-\sum_{j=1}^{N} a_{i j} P_{i}^{-1}\right] P_{i} .
\end{aligned}
$$

Then, the distributed estimate update (27) is directly derived from (31)

Remark 2. The IKCF (24)-(27) is distributed in that the computations of each agent only depend on its neighbors in the graph topology. As such, the computational complexity does not depend on the total number of agents $N$ in the network, but only on the number of neighbors of each agent. Thus, as the network gets bigger, the computational simplicity is retained. Moreover, it is known that the convergence of distributed protocols depends on the network topology, with tree graphs and fully connected graphs (e.g. complete) converging fastest, and graphs with cycles converging slowly Lewis et al. (2014).

Remark 3. The distributed IKCF in Theorem 1 can be implemented in a distributed fashion on the prescribed communication topology only if either: (i) each node knows the target's input $u(t)$, or (ii) $u(t)=0$. This deficiency is corrected in Section 5 by introducing an observer for the target's input $u(t)$.

Remark 4. If there is no communication noise $\omega_{i j}$ between sensor $i$ and sensor $j$, the distributed IKCF (24)(27) can be rewritten as

$$
\begin{aligned}
\dot{P}_{i} & =A_{i} P_{i}+P_{i} A_{i}^{T}+F S F^{T} \\
& -g_{i 0}^{2} P_{i} \bar{H}_{i}^{T} \Omega_{i}^{-1} \bar{H}_{i} P_{i}-P_{i} \sum_{j=1}^{N} a_{i j}\left(P_{j}^{-1}-P_{i}^{-1}\right) P_{i}, \\
\dot{\hat{x}}_{i} & =A \hat{x}_{i}+B u+g_{i 0}^{2} P_{i} \bar{H}_{i}^{T} \Omega_{i}^{-1}\left(\bar{H}_{i}\left(x-\hat{x}_{i}\right)+v_{i}\right) \\
& +P_{i} \sum_{j=1}^{N} a_{i j} P_{j}^{-1}\left(\hat{x}_{j}-\hat{x}_{i}\right) .
\end{aligned}
$$

Comparing these equations to (9) and (10), the summation consensus terms are the same. The first terms in (52) relate to the term $\dot{W}_{i}$ in (9), (10), while the first terms in (53) relate to the term $\dot{z}_{i}$. Comparing these to the work of Olfati-Saber (2007) in (12)-(14), the consensus term in (14) does not have information weighting $P_{j}^{-1}$, so that inaccurate estimates of neighbors are not discounted. Comparing these to the work of Ren et al. (2005) in (16)(18), not only the target's information regarding the terms $g_{i 0}^{2} P_{i} \bar{H}_{i}^{T} \Omega_{i}^{-1} \bar{H}_{i} P_{i}$ and $g_{i 0}^{2} P_{i} \bar{H}_{i}^{T} \Omega_{i}^{-1} \bar{H}_{i}\left(x-\hat{x}_{i}\right)$, but also the target's dynamics including the terms of $A_{i} P_{i}+P_{i} A_{i}^{T}$ and $A \hat{x}_{i}+B u$ are encoded into (52) and (53). Comparing these to the MKCF in Lemma 1 in (20)-(21), the covariances update (52) is different from (20). In fact, the distributed 
information-weighted Kalman consensus filter in Theorem 1 allows for a rigorous convergence analysis as shown formally in next section.

Remark 5. Note that the IKCF incorporates information about measurements of the target state, but also information about the initial estimates of all the nodes. If the node is fully naive, that is, fully uninformed in the sense that it has neither measurements of the target state, but also no initial information, then $g_{i 0}=0$ in (52), (53) (and also of course in (24)-(27)). Moreover, if node $i$ has no initial information and has inputs from only one node $j$, then $P_{i} \rightarrow \infty$ and it is seen from (53) that on convergence one has $\hat{x}_{i} \rightarrow \hat{x}_{j}$. That is, in this case the estimate of node $j$ is simply passed on to node $i$.

\subsection{Stability and convergence analysis of the distributed IKCF}

The information-weighted measurement model (22) results in distributed IKCF in Theorem 1. That structure allows for a formal proof of convergence to consensus estimates of the target's state by all the agents as detailed in the next result. The following results on graph theory are needed.

Lemma 2 (Lewis et al., 2014). The Laplacian matrix $L$ has rank $N$-1, i.e. $\lambda_{1}=0$ is nonrepeated, if and only if the directed graph $G$ has a spanning tree.

Therefore, under Assumption $1 \lambda_{1}=0$ is nonrepeated. Then $L \underline{1} c=0$ with $\underline{1}=\left[\begin{array}{lll}1 & \cdots & 1\end{array}\right]^{T} \in \mathfrak{R}^{N}$ the vector of 1 's and $c$ any constant. Moreover, under Assumption $1, L$ is a singular $\mathrm{M}$ matrix and the following result holds.

Lemma $3(\mathrm{Qu}, 2009)$. Let the Laplacian matrix $L$ be a singular M-matrix. Then there exists a positive vector $q=\left[q_{1}, q_{2}, \cdots, q_{N}\right]^{T}$ with $q_{i}>0 \quad, \forall i=1,2, \ldots, N$ such that $q^{T} L \geq 0$. (A vector is positive if all its elements are positive.)

The next new result extends results in Zhang, Lewis, \& $\mathrm{Qu}$ (2011) and is needed in the proof of Theorem 2.

Lemma 4. Let the Laplacian matrix $L$ be a singular Mmatrix and define $q=\left[q_{1}, q_{2}, \cdots, q_{N}\right]^{T}$ by Lemma 3 . Then $q_{i} \sum_{j=1}^{N} a_{i j} \geq \sum_{j=1}^{N} q_{j} a_{j i} \geq 0, \quad \forall i=1,2 \ldots, N$.

Proof. It is straightforward that

$$
\begin{aligned}
& q^{T} L=\left[q_{1} \sum_{j=1}^{N} a_{1 j}-\sum_{j=1}^{N} q_{j} a_{j 1} \quad q_{2} \sum_{j=1}^{N} a_{2 j}-\sum_{j=1}^{N} q_{j} a_{j 2}\right. \\
& \left.\cdots \quad q_{N} \sum_{j=1}^{N} a_{N j}-\sum_{j=1}^{N} q_{j} a_{j N}\right] \geq 0 .
\end{aligned}
$$

By Lemma 3, all the elements in vector $q^{T} L$ are nonnegative, i.e.

$$
q_{i} \sum_{j=1}^{N} a_{i j}-\sum_{j=1}^{N} q_{j} a_{j i} \geq 0, \quad \forall i=1,2, \ldots N .
$$

Since $q_{i}>0$ and $a_{i j} \geq 0$, it can be easily derived that

$$
q_{i} \sum_{j=1}^{N} a_{i j} \geq \sum_{j=1}^{N} q_{j} a_{j i} \geq 0, \quad \forall i=1,2, \ldots N .
$$

This completes the proof.

Theorem 2: Proof of Convergence to Consensus of the distributed IKCF. Under Assumptions 1 and 2, consider the information flow structure (22) and the associated distributed IKCF (24)-(27). Suppose that process noises $v_{i}(t)$ and channel transmission noises $\omega_{i j}(t)$ are equal to zero. Then the state estimates $\hat{x}_{i}(t)$ achieve consensus and converge to the target's state, i.e., the distributed state estimation error defined as $\tilde{x}_{i}(t)=x(t)-\hat{x}_{i}(t) \in \mathfrak{R}^{n}$ goes to zero as $t \rightarrow \infty$.

Proof. Under Assumption 1, the communication graph has a spanning tree and $g_{i 0}=1$ for a root node $i$. According to the distributed information flow structure (22), define (28) and (29) so that one has the distributed measurement model without noises given by

$$
z_{i}^{d i r}=g_{i 0} \bar{H}_{i} x
$$

and

$$
z_{i}^{\text {ind }}=\sum_{j=1}^{N} a_{i j} P_{j}^{-1} \hat{x}_{j} .
$$

Therefore, the distributed IKCF for the state estimation without noises is given based on (53) as

$$
\begin{aligned}
\dot{\hat{x}}_{i} & =A \hat{x}_{i}+B u+g_{i 0}^{2} P_{i} \bar{H}_{i}^{T} \Omega_{i}^{-1} \bar{H}_{i}\left(x-\hat{x}_{i}\right) \\
& +P_{i} \sum_{j=1}^{N} a_{i j} P_{j}^{-1}\left(\hat{x}_{j}-\hat{x}_{i}\right) \\
= & A_{i} \hat{x}_{i}+B u+g_{i 0}^{2} P_{i} \bar{H}_{i}^{T} \Omega_{i}^{-1} \bar{H}_{i}\left(x-\hat{x}_{i}\right) \\
& +P_{i} \sum_{j=1}^{N} a_{i j} P_{j}^{-1}\left(\hat{x}_{j}-\hat{x}_{i}\right)-\sum_{j=1}^{N} a_{i j} \hat{x}_{i} .
\end{aligned}
$$

Also, the target's dynamics without noise are

$$
\dot{x}=A x+B u \text {. }
$$

The local state synchronization error dynamic and state estimation covariance are derived as

$$
\begin{aligned}
\dot{\tilde{x}}_{i}= & \dot{x}-\dot{\hat{x}} \\
= & A x+B u-A_{i} \hat{x}_{i}-B u-g_{i 0}^{2} P_{i} \bar{H}_{i}^{T} \Omega_{i}^{-1} \bar{H}_{i}\left(x-\hat{x}_{i}\right) \\
& -P_{i} \sum_{j=1}^{N} a_{i j} P_{j}^{-1}\left(\hat{x}_{j}-\hat{x}_{i}\right)-\sum_{j=1}^{N} a_{i j} \hat{x}_{i} \\
& =\left(A_{i}-g_{i 0}^{2} P_{i} \bar{H}_{i}^{T} \Omega_{i}^{-1} \bar{H}_{i}-P_{i} \sum_{j=1}^{N} a_{i j} P_{j}^{-1}\right) \tilde{x}_{i} \\
& +P_{i} \sum_{j=1}^{N} a_{i j}\left(P_{j}^{-1} \tilde{x}_{j}-P_{i}^{-1} \tilde{x}_{i}\right),
\end{aligned}
$$




$$
\begin{aligned}
\dot{P}_{i} & =A_{i} P_{i}+P_{i} A_{i}^{T}+F S F^{T} \\
& -g_{i 0}^{2} P_{i} \bar{H}_{i}^{T} \Omega_{i}^{-1} \bar{H}_{i} P_{i}-P_{i} \sum_{j=1}^{N} a_{i j}\left(P_{j}^{-1}-P_{i}^{-1}\right) P_{i} .
\end{aligned}
$$

Define a Lyapunov function candidate as $V(\tilde{\mathbf{x}})=\sum_{i=1}^{N} q_{i} \tilde{x}_{i}^{T} P_{i}^{-1} \tilde{x}_{i}$ using the vector $q$ defined in Lemma

3. Under Assumption 2, $P=\operatorname{diag}\left(P_{i}^{-1}\right)$ is positive definite if the pair $(A, H)$ is observerable such that $V(\tilde{\mathbf{x}})>0$ for any $\tilde{\mathbf{x}}=\left[\tilde{x}_{1}, \tilde{x}_{2}, \ldots, \tilde{x}_{N}\right]^{T} \neq 0$. Then the one has

$$
\dot{V}(\tilde{\mathbf{x}})=\sum_{i=1}^{N} q_{i} \tilde{x}_{i}^{T} P_{i}^{-1} \dot{\tilde{x}}_{i}+\sum_{i=1}^{N} q_{i} \dot{\tilde{x}}_{i}^{T} P_{i}^{-1} \tilde{x}_{i}-\sum_{i=1}^{N} q_{i} \tilde{x}_{i}^{T} P_{i}^{-1} \dot{P}_{i} P_{i}^{-1} \tilde{x}_{i} .
$$

Substituting (52) and (61) into (63) leads to

$$
\begin{aligned}
\dot{V}(\tilde{\mathbf{x}})= & -\sum_{i=1}^{N} q_{i} \tilde{x}_{i}^{T} P_{i}^{-1} F S F^{T} P_{i}^{-1} \tilde{x}_{i}-2 \sum_{i, j=1}^{N} q_{i} a_{i j} \tilde{x}_{i}^{T} P_{i}^{-1} \tilde{x}_{i} \\
& -\sum_{i=1}^{N} q_{i} g_{i 0}^{2} \tilde{x}_{i}^{T} \bar{H}_{i}^{T} \Omega_{i}^{-1} \bar{H}_{i} \tilde{x}_{i}+\sum_{i=1}^{N} q_{i} \tilde{x}_{i}^{T} \sum_{j=1}^{N} a_{i j} P_{j}^{-1}\left(\tilde{x}_{j}-\tilde{x}_{i}\right) \\
& +\sum_{i, j=1}^{N} q_{i} a_{i j}\left(P_{j}^{-1} \tilde{x}_{j}-P_{i}^{-1} \tilde{x}_{i}\right)^{T} \tilde{x}_{i} .
\end{aligned}
$$

The last three terms at the right side of (64) becomes

$$
\begin{aligned}
& -\sum_{i=1}^{N} q_{i} g_{i 0}^{2} \tilde{x}_{i}^{T} \bar{H}_{i}^{T} \Omega_{i}^{-1} \bar{H}_{i} \tilde{x}_{i}-\sum_{i=1}^{N} q_{i} \tilde{x}_{i}^{T} \sum_{j=1}^{N} a_{i j} P_{j}^{-1}\left(\tilde{x}_{i}-\tilde{x}_{j}\right) \\
& -\sum_{i, j=1}^{N} q_{i} a_{i j}\left(P_{i}^{-1} \tilde{x}_{i}-P_{j}^{-1} \tilde{x}_{j}\right)^{T} \tilde{x}_{i} \\
& =-\tilde{\mathbf{x}}^{T}\left[\left(Q(\tilde{L}+\tilde{G})+(\tilde{L}+\tilde{G})^{T} Q\right)\right] \tilde{\mathbf{x}} \equiv-\tilde{\mathbf{x}}^{T} Q^{*} \tilde{\mathbf{x}}
\end{aligned}
$$

where $Q=\operatorname{diag}\left(q_{i} \otimes I_{n}\right), \tilde{G}=\operatorname{diag}\left(q_{i} g_{i 0}^{2} \bar{H}_{i}^{T} \Omega_{i}^{-1} \bar{H}_{i}\right)$ and

$$
\tilde{L}=\left[\begin{array}{ccc}
\sum_{j=1}^{N} a_{1 j} P_{j}^{-1}-a_{11} P_{1}^{-1} & \ldots & -a_{1 N} P_{N}^{-1} \\
\vdots & \ddots & \vdots \\
-a_{N 1} P_{1}^{-1} & \cdots & \sum_{j=1}^{N} a_{N j} P_{j}^{-1}-a_{N N} P_{N}^{-1}
\end{array}\right] .
$$

According to Lemma 4, the fifth term at the right hand side of $\dot{V}\left(\tilde{x}_{i}\right)$ in (64) becomes

$$
\begin{aligned}
& \sum_{i, j=1}^{N} q_{i} a_{i j}\left(P_{j}^{-1} \tilde{x}_{j}-P_{i}^{-1} \tilde{x}_{i}\right)^{T} \tilde{x}_{i} \\
= & \sum_{i=1}^{N} q_{i} \tilde{x}_{j}^{T} \sum_{j=1}^{N} a_{i j} P_{j}^{-1} \tilde{x}_{i}-\sum_{i=1}^{N} \tilde{x}_{i}^{T} P_{i}^{-1}\left(q_{i} \sum_{j=1}^{N} a_{i j}\right) \tilde{x}_{i} \\
\leq & \sum_{i=1}^{N} q_{i} \tilde{x}_{j}^{T} \sum_{j=1}^{N} a_{i j} P_{j}^{-1} \tilde{x}_{i}-\sum_{i=1}^{N} \tilde{x}_{i}^{T} P_{i}^{-1}\left(\sum_{j=1}^{N} q_{j} a_{j i}\right) \tilde{x}_{i} \\
= & \sum_{i=1}^{N} q_{i} \tilde{x}_{j}^{T} \sum_{j=1}^{N} a_{i j} P_{j}^{-1} \tilde{x}_{i}-\sum_{j=1}^{N} \tilde{x}_{j}^{T} P_{j}^{-1}\left(\sum_{i=1}^{N} q_{i} a_{i j}\right) \tilde{x}_{j} \\
= & \sum_{i, j=1}^{N} q_{i} a_{i j} \tilde{x}_{j}^{T} P_{j}^{-1}\left(\tilde{x}_{i}-\tilde{x}_{j}\right) .
\end{aligned}
$$

According to Lemma 6 in Zhang, Lewis, \& Qu (2011), substituting (66) into (65) leads to

$$
\begin{aligned}
& -\tilde{\mathbf{x}}^{T} Q^{*} \tilde{\mathbf{x}} \leq-\sum_{i=1}^{N} q_{i} g_{i 0}^{2} \tilde{x}_{i}^{T} \bar{H}_{i}^{T} \Omega_{i}^{-1} \bar{H}_{i} \tilde{x}_{i} \\
& -\sum_{i, j=1}^{N} q_{i} a_{i j} \tilde{x}_{i}^{T} P_{j}^{-1}\left(\tilde{x}_{i}-\tilde{x}_{j}\right)-\sum_{i, j=1}^{N} q_{i} a_{i j} \tilde{x}_{j}^{T} P_{j}^{-1}\left(\tilde{x}_{j}-\tilde{x}_{i}\right) \\
& =-\sum_{i=1}^{N} q_{i} g_{i 0}^{2} \tilde{x}_{i}^{T} \bar{H}_{i}^{T} \Omega_{i}^{-1} \bar{H}_{i} \tilde{x}_{i} \\
& -\sum_{i, j=1}^{N} q_{i} a_{i j}\left(\tilde{x}_{j}-\tilde{x}_{i}\right)^{T} P_{j}^{-1}\left(\tilde{x}_{j}-\tilde{x}_{i}\right) \\
& <0 .
\end{aligned}
$$

By concatenating all the equations above, the derivative of the Lyapunov function becomes

$$
\dot{V}(\tilde{\mathbf{x}}) \leq-\tilde{\mathbf{x}}^{T} Q^{*} \tilde{\mathbf{x}}<0 .
$$

Therefore $V(\tilde{\mathbf{x}})$ is a Lyapunov function and state error dynamics (61) is globally asymptotically stable, which implies that all the estimates follow the target's state, i.e. $\tilde{x}_{i}(t)=x(t)-\hat{x}_{i}(t) \rightarrow 0, \forall i$ if there are no process and communication noise.

If the process and communication noise are not zero, it can be shown that the state estimation errors $\tilde{x}_{i}(t)=x(t)-\hat{x}_{i}(t) \in \mathfrak{R}^{n}$ are uniformly ultimately bounded (Lewis et al., 2014) so that the estimated states converge to the target's state within a bounded region whose size depends on the noises.

\section{Information-weighted KCF with unknown target's inputs}

The distributed IKCF in Theorem 1 can be implemented in a distributed fashion on the prescribed communication topology only if either: (i) each node knows the target's input $u(t)$, or (ii) $u(t)=0$. This deficiency is corrected in this section by introducing a distributed observer for the target's input $u(t)$, which can only be directly observed by "informed nodes (sensors)" in application areas.

\subsection{Distributed IKCF for target input estimation}

Assume that the target's input $u(t)$ can be modeled as the output of a continuous-time (CT) linear exosystem, i.e., a command generator, in state-space form as

$$
\dot{u}=A_{u} u+F_{u} w_{u}, \quad u \in \mathfrak{R}^{m}
$$

where $u(0) \sim\left(\bar{u}_{0}, P_{u, 0}\right)$ and noise $w_{u} \sim\left(0, S_{u}\right)$.

Then, analogous to the case of the state measurement model (22) in Section 4, an information-weighted distributed measurement model for the target's input of the $i$-th sensor can be taken as

$$
z_{u, i}=\left(\begin{array}{c}
g_{i 0}\left(\bar{H}_{u, i} u+v_{u, i}\right) \\
\sum_{j=1}^{N} a_{i j} P_{u, j}^{-1}\left(u_{j}+\omega_{u, i j}\right)
\end{array}\right) \equiv H_{u, i} u+v_{u, i}
$$

where $\bar{H}_{u, i}$ are measurement observation matrices, 
$P_{u, i}=E\left\{\left(u_{i}-u\right)\left(u_{i}-u\right)^{T}\right\} \in \mathfrak{R}^{m \times m}$ is the input estimation error covariance matrices, process noise of the target's input is $v_{u, i}(t)$ and channel transmission noises of the target's input are $\omega_{u, i j}(t)$. Assuming the target's input can be observed by the same nodes that can directly observe the target's state in (22), the pinning gains $g_{i 0}$ in (70) are the same as those in (22).

The information flow structure of the target's input (70) can be written in the standard form (11) with

$H_{u, i}=\left(\begin{array}{c}g_{i 0} \bar{H}_{u, i} \\ \sum_{j=1}^{N} a_{i j} P_{u, j}^{-1}\end{array}\right), \quad v_{u, i}=\left(\begin{array}{c}g_{i 0} v_{u, i} \\ \sum_{j=1}^{N} a_{i j} P_{u, j}^{-1}\left(u_{j}-u+\omega_{u, i j}\right)\end{array}\right)$.

Now, Theorem 1 can be applied to the target's generator (69) with distributed measurements (70) to write the distributed IKCF for estimating the target's input.

$$
\begin{aligned}
\dot{P}_{u, i}= & A_{u, i} P_{u, i}+P_{u, i} A_{u, i}^{T}+F_{u} S_{u} F_{u}^{T} \\
& +\sum_{j=1}^{N} a_{i j} P_{u, i} B^{T} P_{i}^{-1} B P_{u, i}-g_{i 0}^{2} P_{u, i} \bar{H}_{u, i}^{T} \Omega_{u, i}^{-1} \bar{H}_{u, i} P_{u, i} \\
& -P_{u, i}\left[\left(\sum_{j=1}^{N} a_{i j} P_{u, j}^{-1}\right)\left(\sum_{j=1}^{N} a_{i j}\left(P_{u, j}^{-1}+P_{u, j}^{-1} \Pi_{u, i j} P_{u, j}^{-1}\right)\right)^{-1}\right. \\
& \left.\cdot\left(\sum_{j=1}^{N} a_{i j} P_{u, j}^{-1}\right)-\sum_{j=1}^{N} a_{i j} P_{u, i}^{-1}\right] P_{u, i}, \\
K_{u, i}^{d i r} & =g_{i 0} P_{u, i} \bar{H}_{u, i}^{T} \Omega_{u, i}^{-1}, \\
K_{u, i}^{i n d} & =P_{u, i}\left(\sum_{j=1}^{N} a_{i j} P_{u, j}^{-1}\right)\left(\sum_{j=1}^{N} a_{i j}\left(P_{u, j}^{-1}+P_{u, j}^{-1} \Pi_{u, i j} P_{u, j}^{-1}\right)\right)^{-1}, \\
\dot{u}_{i}= & A_{u} u_{i}+K_{u, i}^{d i r}\left(g_{i 0} \bar{H}_{u, i}\left(u-u_{i}\right)+v_{u, i}\right) \\
& +K_{u, i}^{i n d} \sum_{j=1}^{N} a_{i j} P_{u, j}^{-1}\left(u_{j}-u_{i}+\omega_{u, i j}\right)
\end{aligned}
$$

where $\quad A_{u, i}=A_{u}+\sum_{j=1}^{N} a_{i j} I_{n} \quad, \quad \Omega_{u, i}=E\left\{g_{i 0}^{2} v_{u, i} v_{u, i}^{T}\right\}$, $\Pi_{u, i j}=E\left\{\omega_{u, i j} \omega_{u, i j}^{T}\right\}$ for all $i, j=1,2, \ldots N$.

Therefore, the distributed IKCF for a target with unknown input is given by the next result.

Theorem 3: Distributed IKCF driven by an unknown target's input. Consider the target's state equation (1) with the distributed information flow structure (22), and the target's input generator (69) with the distributed information flow structure (70). Then, the standard CTKF equations (3)-(5) can be written as the distributed IKCF with the covariance propagation equations for target's state (24) and for target's input (72), the direct and indirect Kalman gains for target's state (25)-(26) and for target's input (73)-(74), and the distributed agent estimate updates for target's input (75) and for target's state (27) replaced by

$$
\begin{aligned}
\dot{\hat{x}}_{i} & =A \hat{x}_{i}+B u_{i}+g_{i 0} K_{i}^{\text {dir }}\left(\bar{H}_{i}\left(x-\hat{x}_{i}\right)+v_{i}\right) \\
& +K_{i}^{i n d} \sum_{j=1}^{N} a_{i j} P_{j}^{-1}\left(\hat{x}_{j}-\hat{x}_{i}+\omega_{i j}\right) .
\end{aligned}
$$

Proof. Since the corresponding proofs follow the same lines of argument from Theorem 1, they are omitted here.

\subsection{Stability and convergence analysis of IKCF with un known target's input}

The stability and convergence analysis of the distributed IKCF in Theorem 3 for state estimation of the target's dynamics (1) driven by an unknown target's input $u(t)$ are summarized in the following Theorem.

Theorem 4: Proof of Convergence to Consensus of IKCF with target's input unknown. Under Assumption 1 and 2, consider the information flow structure (22) for the state estimates and (70) for the target's input estimates and the associated distributed IKCF (24)-(26) and (72)-(76). Suppose that process noises $v_{i}(t)$ and $v_{u, i}(t)$ and channel transmission noises $\omega_{i j}(t)$ and $\omega_{u, i j}(t)$ are equal to zero. Then the state estimates $\hat{x}_{i}(t)$ achieve consensus and converge to the target's state, i.e., the distributed state estimation error defined as $\tilde{x}_{i}(t)=x(t)-\hat{x}_{i}(t) \in \mathfrak{R}^{n}$ goes to zero as $t \rightarrow \infty$, and the target's input estimates $u_{i}(t)$ achieve consensus and converge to the target's input, i.e., the distributed input estimation error defined as $\tilde{u}_{i}(t)=u(t)-u_{i}(t) \in \mathfrak{R}^{m}$ goes to zero as $t \rightarrow \infty$.

Proof. Under Assumption 1, the communication graph has a spanning tree and $g_{i 0}=1$ for a root node $i$. According to (61) and (76), the local state synchronization error dynamics without noises are

$$
\begin{aligned}
\dot{\tilde{x}}_{i} & =\left(A_{i}-g_{i 0}^{2} P_{i} \bar{H}_{i}^{T} \Omega_{i}^{-1} \bar{H}_{i}-P_{i} \sum_{j=1}^{N} a_{i j} P_{j}^{-1}\right) \tilde{x}_{i}+B \tilde{u}_{i} \\
& +P_{i} \sum_{j=1}^{N} a_{i j}\left(P_{j}^{-1} \tilde{x}_{j}-P_{i}^{-1} \tilde{x}_{i}\right) .
\end{aligned}
$$

According to (69) and (75), the local input synchronization error dynamics without noises are

$$
\begin{aligned}
\dot{\tilde{u}}_{i} & =\left(A_{u, i}-g_{i 0}^{2} P_{u, i} \bar{H}_{u, i}^{T} \Omega_{u, i}^{-1} \bar{H}_{u, i}-P_{u, i} \sum_{j=1}^{N} a_{i j} P_{u, j}^{-1}\right) \tilde{u}_{i} \\
& +P_{u, i} \sum_{j=1}^{N} a_{i j}\left(P_{u, j}^{-1} u_{j}-P_{u, i}^{-1} u_{i}\right) .
\end{aligned}
$$

Define a new Lyapunov function candidate as $V(\tilde{\mathbf{x}}, \tilde{\mathbf{u}})=\sum_{i=1}^{N} q_{i}\left(\tilde{x}_{i}^{T} P_{i}^{-1} \tilde{x}_{i}+\tilde{u}_{i}^{T} P_{u, i}^{-1} \tilde{u}_{i}\right) \quad$. By calculating $\dot{V}(\tilde{\mathbf{x}}, \tilde{\mathbf{u}})$ based on (68), (77) and (78) as derived in Section 4.3 , a simplified form are derived as, 


$$
\begin{aligned}
& \dot{V}(\tilde{\mathbf{x}}, \tilde{\mathbf{u}})<-2 \sum_{i, j=1}^{N} q_{i} a_{i j} \tilde{x}_{i}^{T} P_{i}^{-1} \tilde{x}_{i}+\sum_{i=1}^{N} q_{i} \tilde{x}_{i}^{T} P_{i}^{-1} B \tilde{u}_{i} \\
&+\sum_{i=1}^{N} q_{i} \tilde{u}_{i}^{T} B^{T} P_{i}^{-1} \tilde{x}_{i}-\sum_{i, j=1}^{N} q_{i} a_{i j} \tilde{u}_{i}^{T} B^{T} P_{i}^{-1} B \tilde{u}_{i} \\
&=-\sum_{i=1}^{N} q_{i}\left(\tilde{x}_{i} \tilde{u}_{i}\right)^{T} M_{i}\left(\begin{array}{c}
\tilde{x}_{i} \\
\tilde{u}_{i}
\end{array}\right) \\
& \text { where } M_{i}=\left[\begin{array}{cc}
2 \sum_{j=1}^{N} a_{i j} P_{i}^{-1} & -P_{i}^{-1} B \\
-B^{T} P_{i}^{-1} & \sum_{j=1}^{N} a_{i j} B^{T} P_{i}^{-1} B
\end{array}\right] .
\end{aligned}
$$

Matrix $M_{i}$ is positive definite if and only if $2 \sum_{j=1}^{N} a_{i j} P_{i}^{-1}$ is positive definite and the Schur complement defined in Ouellette (1981) of the block $2 \sum_{j=1}^{N} a_{i j} P_{i}^{-1}$ of the matrix $M_{i}$ is positive definite. Since $2 \sum_{j=1}^{N} a_{i j} P_{i}^{-1}$ and the its Schur complement matrix

$$
\begin{aligned}
& Q_{i}=\sum_{j=1}^{N} a_{i j} B^{T} P_{i}^{-1} B-B^{T} P_{i}^{-1}\left(2 \sum_{j=1}^{N} a_{i j} P_{i}^{-1}\right)^{-1} P_{i}^{-1} B \\
& =\frac{1}{2} \sum_{j=1}^{N} a_{i j} B^{T} P_{i}^{-1} B
\end{aligned}
$$

are both positive definite matrices, $M_{i}$ is a positive definite matrix, therefore

$$
\dot{V}(\tilde{\mathbf{x}}, \tilde{\mathbf{u}})<0 \text {. }
$$

Both state and input error dynamics of sensor networks are globally asymptotically stable by using the proposed distributed IKCF if there is no noise. That is to say the consensus or synchronization to a target or leader node can be guaranteed without bias by employing pinned measurement correction term and weighted average consensus regulator.

\section{Illustrative example}

It is shown in this section that the new IKCF in Theorem 1 outperforms the KCF in Olfati-Saber (2007) and the MKCF in Ren et al. (2005). To evaluate the performance of the proposed distributed IKCF, this section presents simulation results for a sensor network as shown in Fig.1.

Node 0 is the "target node" and has the dynamics (1). The state of node 0 is known as the target state whose value is to be estimated by all the six sensors labeled from 1 to 6 . Node 1 and 4 are known as "informed nodes" and have direct measurements of the target node 0 . Node 2 and 3 can only receive the information about target state through node 1 and 4 indirectly. Neither node 5 and 6 nor their immediate neighbor node 2 receive direct measurements from the target node 0 . Thus node 5 and 6 are known as "naive nodes" due to their limited observability.

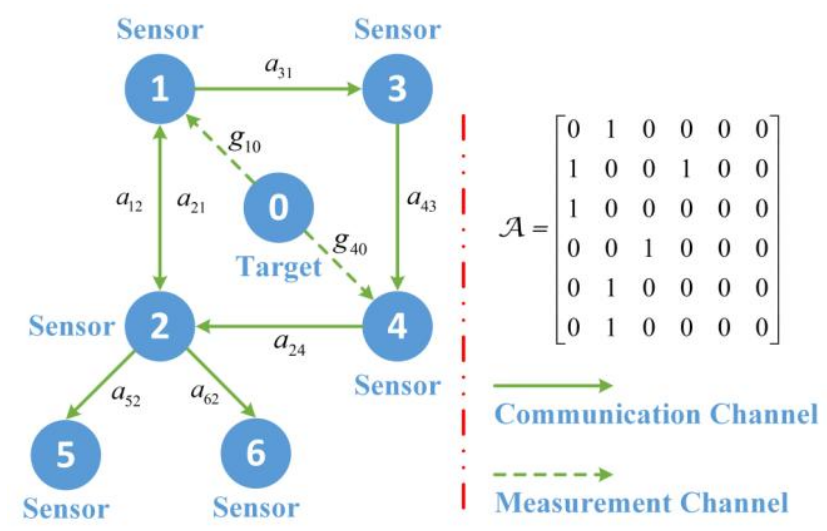

Fig. 1. The network topology, with target node 0 .

\subsection{Performance with zero target's input}

First, the proposed distributed IKCF (24)-(27) is verified. We consider the communication graph in Fig. 1. Let the system dynamics and sensing model be linear time invariant as shown in (1), (28) and (29) with zero input, where the state vector $x(t), \hat{x}_{i}(t) \in \mathfrak{R}^{4}$ consist of two position and two velocity components. A boundary stabilization system is adopted here, namely,

$A=\left[\begin{array}{cccc}0 & -0.5 & 0 & 0 \\ 0.5 & 0 & 0 & 0 \\ 0 & 0 & 0 & 0.5 \\ 0 & 0 & -0.5 & 0\end{array}\right]$.

Also, the process, measurement and communication noise are selected as $w=\operatorname{randn}(1) *[2,2,1,1]^{T}$, $v_{i}=0.5 * \operatorname{randn}(4,6)$ and $\omega_{i j}=0.5 * \operatorname{randn}(4,6,6)$. Other settings used for this revised dynamical model are as follows: $F=I_{4}, \quad S=\operatorname{diag}\{2,2,1,1\} \quad, \quad \Omega_{i}=0.5 * I_{4}$, $\Pi_{i j}=0.5 * I_{4}$ for $i, j=1,2, \ldots 6$, where $I_{n}$ represents an $n \times n$ identity matrix. The initial states and covariance matrices of target and sensors are picked from 0-5 and 0-3, respectively.

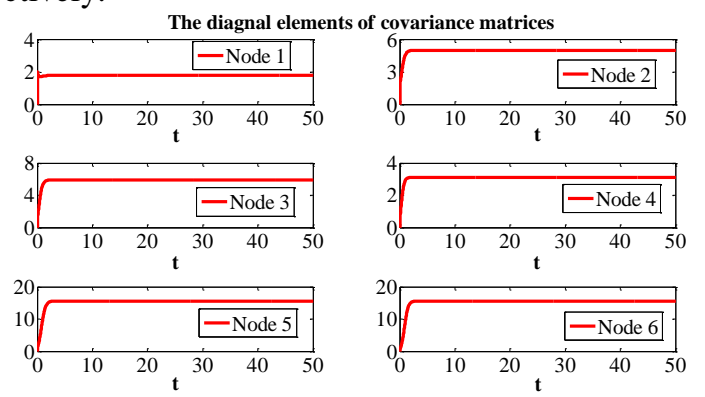

Fig 2. The diagnal elements $P_{22}$ of covariance matrices of six nodes

The diagonal element $P_{22}$ of the covariance matrix of six nodes are illustrated in Fig. 2. It can be seen that Node 1 
and 4 has smaller $P_{22}$ than other nodes when it can directly obtain the target's state information. Otherwise, the $5^{\text {th }}$ and $6^{\text {th }}$ agents' estimates are discounted by their large covariance $P_{22}$. Fig. 3 gives the tracking state error by using (24)-(27). The state estimation errors $\tilde{x}_{i}(t)=x(t)-\hat{x}_{i}(t)$ are uniformly ultimately bounded in the presence of process noise and communication noise. For the comparison of different KCF methods, two kinds of quantitative evaluations of the tracking state error are given later by Table 1 and 2 in Section 6.2, respectively.
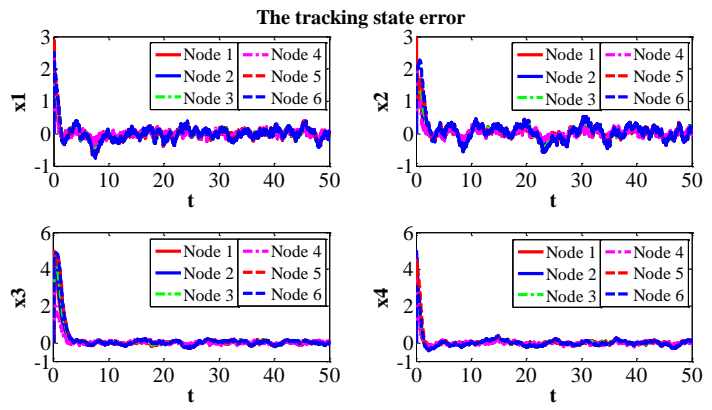

Fig. 3. The tracking state error by using (24)-(27)

\subsection{Comparison between different KCF methods with ze- ro target's input}

Now, the new IKCF (24)-(27) is compared with two existing methods, the distributed KCF (12)-(14) from Olfati-Saber (2007) and the distributed MKCF (20)-(21) from Ren et al. (2005). Also, two kinds of evaluation methods are used to evaluate the performance of three $\mathrm{KCF}$ methods from the point of view of deterministic and stochastic systems.

The special settings for the distributed KCF (12)-(14) are given by $H_{i}=I_{4}$ for those sensors who can observer the target's state and $\gamma=\frac{\varepsilon}{1+\left\|P_{i}\right\|_{F}}>0$ with $\varepsilon=0.01$ as detailed in Olfati-Saber (2009). Other settings are still borrowed from the section 6.1 .
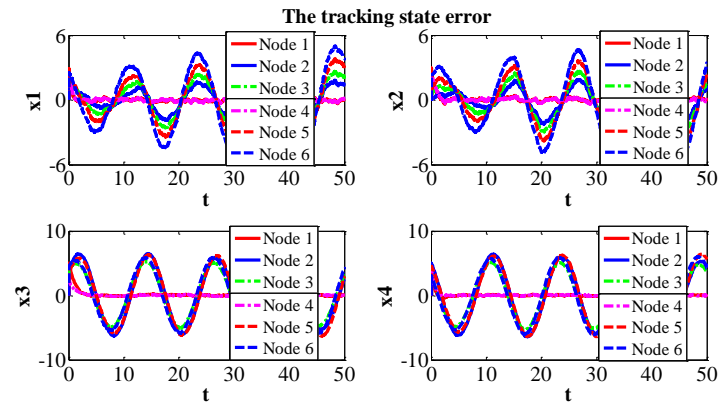

Fig. 4. The tracking state error by using (12)-(14)

Compared with those two methods, the proposed distributed IKCF (24)-(27) performs better obviously, as illustrated in Fig. 3, 4 and 5. From Fig. 4, it is hard to say the distributed KCF (12)-(14) can achieve consensus and guarantee the final consensus value converge to the target's state under the challenging "Tree" topology with a loop. Since there are no information-weighted matrices $P_{j}^{-1}(t)$ in front of consensus terms, the bad estimates from naive nodes diverge the performance. This results are consistent with the theory analysis. Although an acceptable convergence are achieved by using the distributed $\mathrm{MKCF}$ (20)-(21) in Fig. 5, the proposed distributed IKCF (24)(27) has a faster convergence rate and a smaller MSE as shown in Fig. 3.
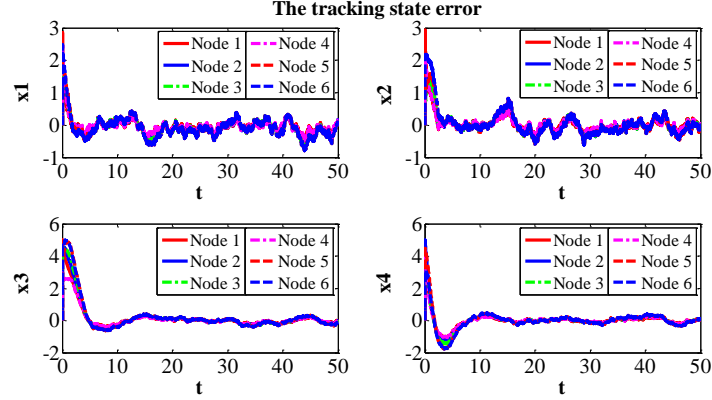

Fig. 5. The tracking state error by using (20)-(21)

The Mean Squared Error (MSE) of the tracking state error is first calculated from the deterministic system perspective. The MSE of all the states of estimates for each sensor during the time interval $[0, T]$ is defined as $M S E_{i}=\frac{1}{T} \int_{0}^{T}\left(x(\tau)-\hat{x}_{i}(\tau)\right)^{2} d \tau \quad$ where $\quad x(t), \hat{x}_{i}(t) \in \mathfrak{R}^{4}$. The MSE of all the sensors is defined as $M S E=\frac{1}{N} \sum_{i=1}^{N} M S E_{i}$. The $M S E$ by using three methods are given by Table 1 . The MSE by using the distributed IKCF (24)-(27) have an decrease of $97.8 \%$ of that by using the distributed $\mathrm{KCF}$ (12)-(14) and an decrease of $52.1 \%$ of that by using the distributed MKCF (20)-(21).

Table 1. The MSE by using three methods

\begin{tabular}{ll}
\hline Method & MSE \\
\hline The distributed KCF (12)-(14) & 7.2053 \\
The distributed MKCF (20)-(21) & 0.3248 \\
The distributed IKCF (24)-(27) & 0.1557
\end{tabular}

Moreover, for the comparison of three KCF methods in detail, the mean, variance and confidence interval (CI) of the tracking state error are further employed to analyze the performance from the stochastic system perspective. For the linear system, since both $w$ and $v_{i}$ are zero-mean WGN, the tracking state error $\tilde{x}_{i}$ has a normal distribution, which is considered to be a population. We choose the tracking errors of state $x_{2}$ of informed node 1 and naive node 6 as representative samples. Having obtained a random observations of size 5000 for each sample, the means and variances are calculated and summarized in Table 2. 
Based on the interval estimate for a normal distribution, we calculate the $95 \%$ CIs simultaneous for the difference in Table 2.
As shown in Table 2, the proposed distributed IKCF (24)-(27) has smaller sample mean, variance and narrower 95\% CI than the other two methods. It is worth noting that the KCF (12)-(14) performs better than the MKCF (20)(21) for informed node 1, but fails for the naive node 6 .

Table 2. Summary results for the tracking error of state $x_{2}$ by using three methods

\begin{tabular}{lcccccc}
\hline Method & \multicolumn{2}{c}{ Sample Mean } & Sample variance & \multicolumn{2}{c}{ 95\% CI } \\
\cline { 2 - 7 } & Node 1 & Node 6 & Node 1 & Node 6 & Node 1 & Node 6 \\
\hline The distributed KCF (12)-(14) & 0.0260 & 0.1856 & 0.0800 & 2.4834 & $(0.0182,0.0338)$ & $(0.1419,0.2293)$ \\
The distributed MKCF (20)-(21) & 0.0561 & 0.1176 & 0.0961 & 0.1950 & $(0.0475,0.0647)$ & $(0.1054,0.1298)$ \\
The distributed IKCF (24)-(27) & 0.0172 & 0.0582 & 0.0462 & 0.1455 & $(0.0112,0.0232)$ & $(0.0476,0.0688)$ \\
\hline
\end{tabular}

\subsection{Performance with unknown target's input}

Finally, the proposed distributed IKCF is verified of incorporating estimates the target's unknown input. Consider the command generator (69) with $A_{u}=A, F_{u}=I_{4}$. Other settings are the same as in Section 6.1. The proposed distributed IKCF driven by an unknown target's input for synchronization on state estimation is also applied in "Tree" topology. The tracking state error by using (24)-(26) and (72)-(76) are drawn in Fig. 6. The distributed estimated states driven by unknown input converge to the target's state within a bounded region whose size depends on the process and channel noises.
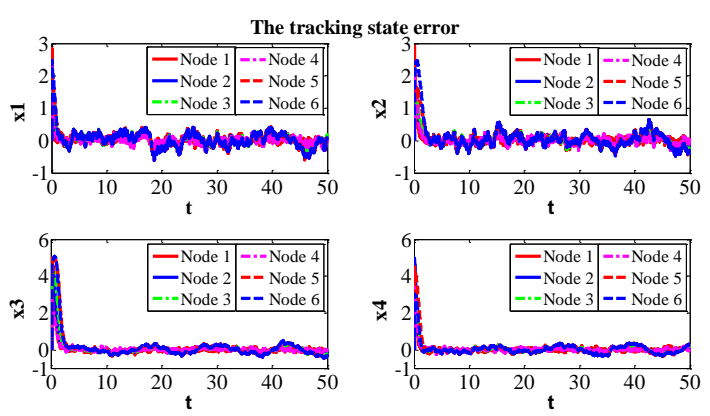

Fig 6. The tracking state error by using (24)-(26) and (72)-(76)

\section{Conclusions}

This paper investigated the distributed IKCF in continuous-time. Based on local information available at each node considering communication topology, a novel networked measurement model was proposed to derive locally optimal solutions for distributed IKCF. Then a formal derivation of convergence was presented by using Lyapunov stability analysis. It was proven that this distributed IKCF was applicable for a dynamic state estimation driven by both known and unknown target's control input even if in the presence of naivety. It was further illustrated in simulation that this distributed IKCF has a better performance than other continuous-time KCFs.
A discrete-time distributed IKCF under multiple targets case will be our future work. Moreover, since the proof of this work is established in a deterministic framework, some stochastic techniques for $\mathrm{KCF}$ such as the convergence of probabilities by using central limit theorem or the law of large numbers are still open issues.

\section{References}

Alighanbari, M., \& How, J. P. (2006). An unbiased Kalman consensus algorithm. In Proceeding of American Control Conference (pp. 3519-3524).

Bidram A., Lewis F. L., \& Davoudi A. (2014). Distributed control systems for small-scale power networks using multi-agent cooperative control theory. IEEE Control Systems Magazine, 34(6), 56-77.

Crassidis, J., \& Junkins, J. (2004). Optimal Estimation of Dynamic Systems. Boca Raton: CRC Press.

Fax J. A., \& Murray R. M. (2004). Information flow and cooperative control of vehicle formations. IEEE Transactions on Automatic Control, 49(9), 1465-1476.

Ferguson, P., \& How, J. P. (2005). Decentralized estimation algorithms for formation flying spacecraft. In Proceeding of AIAA Guidance, Navigation and Control Conference and Exhibit.

Hashemipour, H. R., Roy S., \& Laub, A. J. (1988). Decentralized structures for parallel Kalman filtering. IEEE Transactions on Automatic Control, 33(1), 88-94.

Jadbabaie, A., Lin J., \& Morse A. S. (2003). Coordination of groups of mobile autonomous agents using nearest neighbor rules. IEEE Transactions on Automatic Control, 48(6), 988-1001.

Kamal, A. T., Ding, C., Song, B., Farrell, J. A., \& Roy-Chowdhury, A. K. (2011). A generalized Kalman consensus filter for wide area video networks. In Proceeding of 50th IEEE Conference on Decision and Control (pp. 7863-7869).

Kamal, A. T., Farrell, J. A., \& Roy-Chowdhury A. K. (2012). Information weighted consensus. In Proceeding of 51th IEEE Conference on Decision and Control (pp. 2732-2737).

Kamal, A. T., Farrell, J. A., \& Roy-Chowdhury A. K. (2013). Information weighted consensus filters and their application in distributed camera networks. IEEE Transactions on Automatic Control, 58(12), 3112-3125.

Lewis, F. L., Xie, L. H., \& Popa, D. (2008). Optimal and robust estimation with an introduction to stochastic control theory. Boca Raton: CRC Press.

Lewis, F. L., Zhang, H. W., Movric, K. H., \& Das, A. (2014). Cooperative control of multi agent system: optimal and adaptive design approaches. London, UK: Springer-Verlag.

Olfati-Saber, R., \& Murray R. M. (2004). Consensus problems in networks of agents with switching topology and time-delays. IEEE Transactions on Automatic Control, 49(9), 1520-1533. 
Olfati-Saber, R. (2005). Distributed Kalman filter with embedded consensus filters. In Proceeding of 44th IEEE Conference on Decision and Control and 2005 European Control Conference (CDC-ECC '05) (pp. 8179-8184).

Olfati-Saber, R., \& Shamma, J. S. (2005). Consensus filters for sensor networks and distributed sensor fusion. In Proceeding of 44th IEEE Conference on Decision and Control and 2005 European Control Conference (CDC-ECC '05) (pp. 6698-6703).

Olfati-Saber, R. (2007). Distributed Kalman filtering for sensor networks. In Proceeding of 46th IEEE Conference on Decision and Control (pp. 5492-5498).

Olfati-Saber, R. (2009). Kalman-consensus filter: Optimality, stability, and performance. In Proceeding of 48th IEEE Conference on Decision and Control (pp. 7036-7042).

Ouellette, D. V. (1981). Schur complement and statistics. Linear Algebra and its Applications, 36, 187-295.

$\mathrm{Qu}$, Zhihua (2009). Cooperative control of dynamical systems: applications to autonomous vehicles. London: Springer-Verlag.

Rao, B. S., \& Durrant-Whyte, H. F. (1991). Fully decentralized algorithm for multisensor Kalman filtering. In Proceedings of IEE Control Theory Application, 138(5), 413-420.

Rao, B. S. Y., Durrant-Whyte, H. F., \& Sheen, J. A. (1993). A fully decentralized multi-sensor system for tracking and surveillance. International Journal of Robotics Research, 12(1), 20-44.

Ren, W., Beard, A. W., \& Kingston, D. B. (2005). Multi-agent Kalman consensus with relative uncertainty. In Proceeding of American Control Conference (pp. 1865-1870).

Sahoo S. R., \& Banavar R. N. (2014). Attitude synchronization of satellites with internal actuation. European Journal of Control, 20(3), 152-161.

Spanos, D. P., Olfati-Saber, R., \& Murray, R. M. (2005a). Distributed sensor fusion using dynamic consensus. In Proceeding of 16th IFAC World Congress. Prague, Czech.

Spanos, D. P., Olfati-Saber, R., \& Murray, R. M. (2005b). Dynamic Consensus on Mobile Networks," In Proceeding of 16th IFAC World Congress. Prague, Czech.

Spanos, D. P., Olfati-Saber, R., \& Murray, R. M. (2005c). Approximate distributed Kalman filtering in sensor networks with quantifiable performance. In Proceeding of 4th International Conference on Information Processing in Sensor Networks (pp. 133139). California, USA.

Strogatz, S. H., \& Stewart, I. (1993). Coupled oscillators and biological synchronization. Scientific American. 269(6), 102-109.

Taj, M., \& Cavallaro, A. (2011). Distributed and decentralized multicamera tracking. IEEE Signal Processing Magazine, 28(3), 4658.

Wang, S. C., Ren, W., \& Li, Z. K. (2014). Information-driven fully distributed Kalman filter for sensor networks in presence of naive nodes. arXiv: 1410.0411.

Wasserman, S., \& Faust, K. (1994) Social network analysis: methods and applications. Cambridge, UK: Cambridge University Press.

Xiao, L., Boyd, S., \& Lai, S. (2006) A space-time diffusion scheme for peer-to-peer least-squares estimation. In Proceeding of 5th International Conference on Information Processing in Sensor Networks (pp. 168-176). Nashville, TN.

Zhang, H. W., Lewis, F. L., \& Qu, Z. H. (2011). Lyapunov, adaptive, and optimal design techniques for cooperative systems on directed communication graphs. IEEE Transactions on Industrial Electronics, 59(7), 3026-3041. 